Recepción: 16 / 04 / 2018

Aceptación: 21 / 06 / 2018

Publicación: 02 / 07 / 2018

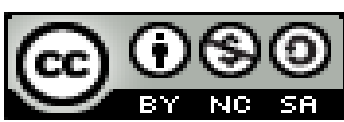

Ciencias económicas y empresariales Artículo de Investigación

\title{
Análisis de la administración de los recursos humanos como parte de la efectiva gestión empresarial
}

Analysis of the management of human resources as part of the effectiveness of business management

Análise da administração de recursos humanos como parte de uma gestão eficaz de negócios

\author{
Milton M. Cárdenas ${ }^{\mathrm{I}}$ \\ milton.cardenas@utc.edu.ec \\ Efrén G. Montenegro-Cueva ${ }^{\mathrm{II}}$ \\ efren.montenegro@utc.edu.ec \\ Nancy F. Moreano-Terán ${ }^{\text {III }}$ \\ nancy.moreano@utc.edu.ec
}

Correspondencia: milton.cardenas@utc.edu.ec

\footnotetext{
${ }^{\text {I } M a g i s t e r ~ e n ~ A d m i n i s t r a c i o ́ n ~ y ~ M a r k e t i n g, ~ D i p l o m a ~ S u p e r i o r ~ e n ~ G e s t i o ́ n ~ A d m i n i s t r a t i v a, ~ E s p e c i a l i s t a ~ e n ~ G e s t i o ́ n ~ d e ~}$ Marketing, Ingeniero Comercial, Docente de la Universidad Técnica de Cotopaxi, Latacunga, Ecuador.

${ }^{\text {II }}$ Magister en Riesgo Financiero, Ingeniero Comercial, Docente de la Universidad Técnica de Cotopaxi, Latacunga, Ecuador.

III Magister en Gestión de la Producción, Ingeniera Agroindustrial, Docente de la Universidad Técnica de Cotopaxi, Latacunga, Ecuador.
} 


\section{Resumen}

Este trabajo sintetiza un estudio realizado en el análisis de la administración de recursos humanos, y la influencia que tiene la relación que existe entre los empleados sobre la efectiva gestión empresarial, a través del estudio de las ramas de la administración y la relación que se tiene entre los departamentos de toda organización. El trabajo hace mención a la influencia que existe sobre la rentabilidad y el cumplimiento de las metas y objetivos, planteados por toda organización en base a la armonía entre los trabajadores. En el estudio se observa que aquellos negocios en donde se tiene mejor calidad humana, los resultados de negocio son más positivos, tanto el entorno como la organización y los individuos están relacionados entre sí y que de esto depende la efectividad de la empresa, para lo que se plantea una gestión estratégica entre los procesos de la administración de recursos humanos. También se estudia el proceso de evolución que ha existido en los departamentos de recursos humanos, desde una visión más abierta y en donde se tiene que la armonía entre el personal es la pieza fundamental para impulsar el crecimiento de la empresa y que se cumpla con los objetivos por la cual se creó, intentando siempre buscando superar sus propias expectativas.

Palabras clave: administración; efectividad; recursos humanos; personal.

\section{Abstract}

This work synthesized a study carried out in the analysis of the administration of human resources and the influence of the relationship that exists between employees on the effectiveness of business management. Through the study of the branches of administration and the relationship that exists between departments throughout the organization. The work has mention of the influence that exists on the profitability and the fulfillment of the goals and objectives raised by the entire organization in the base of the harmony among the workers. The study shows businesses where you have the best quality, the results are more positive, both the environment and the organization and individuals are related to each other and that depends on the effectiveness of the company for what is considered a management strategic between the processes of the administration of human resources. The process of evolution that exists in the human resources departments was also found from a more open view and where there is harmony among the personnel in the fundamental piece 
for the growth of the whole company and that can be seen with the objectives by what are you looking for.

Keywords: administration; effectiveness; human resources; personnel.

\section{Resumo}

Este trabalho sintetiza um estudo realizado na análise da administração de recursos humanos, e a influência da relação existente entre os empregados na gestão eficaz do negócio, através do estudo dos ramos da administração e da relação existente. entre os departamentos de qualquer organização. O trabalho faz menção à influência que existe na lucratividade e no cumprimento das metas e objetivos, levantados por toda organização com base na harmonia entre os trabalhadores. No estudo observa-se que aqueles negócios onde há melhor qualidade humana, os resultados do negócio são mais positivos, tanto o ambiente e a organização quanto os indivíduos estão relacionados entre si e isso depende da efetividade da empresa, por exemplo. que uma gestão estratégica entre os processos de administração de recursos humanos é proposta. O processo de evolução que existe nos departamentos de recursos humanos também é estudado, a partir de uma visão mais aberta e onde a harmonia entre o pessoal é a peça fundamental para promover o crescimento da empresa e que a objetivos para os quais foi criado, sempre tentando superar suas próprias expectativas.

Palavras chave: administração; efetividade; recursos humanos; pessoal.

\section{Introducción}

La administración, es un tema que abarca los puntos más importantes en toda organización, el buen desempeño de las funciones de una empresa depende del cumpliendo de los objetivos generales y específicos y de la efectividad que existe en la gestión empresarial; esta es una de las principales funciones de la administración, organizar y mantener la disciplina dentro de las actividades que se ejecutan en toda empresa.

La ciencia administrativa, sin embargo, se divide en varias ramas entre las que se encuentra recursos humanos; esta área de la administración estudia las relaciones entre las personas que trabajan en una empresa y la manera de cómo mantener en armonía al personal con la finalidad de alcanzar una efectiva gestión en el desarrollo de los procesos que hacen posible la existencia de la empresa. 
La expresión "recursos humanos" se refiere a las personas que forman parte de las organizaciones y que desempeñan en ellas determinadas funciones. Las personas pasan gran parte de su tiempo trabajando en las organizaciones. Éstas necesitan a las personas para sus actividades y operaciones, de la misma manera que necesitan recursos financieros, materiales y tecnológicos. De ahí la denominación de recursos humanos para describir a las personas que trabajan en las organizaciones. (Chiavenato, 2007, p. 2)

La administración de recursos humanos se mueve en un contexto formado por organizaciones y personas. Administrar personas significa trabajar con las personas que forman parte de las organizaciones. Más aún, significa administrar los demás recursos con las personas. Así las organizaciones y las personas constituyen la base fundamental sobre la que se mueve la ARH. (Administración de Recursos Humanos) (Chiavenato, 2007, p. 7)

La efectividad que existe en toda organización, depende del personal que maneja las gestiones empresariales, mientras mejor sea el trato entre las personas se verá reflejado una mayor eficiencia en los procesos, ya que, estos están interrelacionados, por lo tanto, mantener la armonía entre el personal es la clave principal del departamento de recursos humanos.

El análisis de la administración de recursos humanos es tarea primordial en toda empresa, y debería ser uno de los temas más importantes. Las organizaciones lo que buscan es un mayor crecimiento, una mayor productividad y satisfacer cada día más las necesidades de sus consumidores, a través del cumplimiento de los objetivos generales y específicos, superando sus propias expectativas. Para lograrlo, se necesita que exista una mayor armonía y buen trato entre las personas que conforman la organización.

El objetivo general de la administración de Recursos Humanos, es el mejoramiento del desempeño y de las aportaciones del personal a la organización, en el marco de una actividad ética y socialmente responsable. Este objetivo guía el estudio de la Administración de Recursos Humanos, el cual describe las acciones que pueden y deben llevar a cabo los administradores de esta área. (Peña, 2012).

Estas acciones deben estar bien definidas para cada personal, a modo de mantener en claro las funciones de cada uno en sus áreas y las actividades a desempeñarse sin entorpecer las relaciones entre las personas ni las responsabilidades de cada miembro del departamento. A través de la 
administración de recursos humanos se busca organizar y maximizar el desempeño y productividad de los empleados.

Este departamento es el encargado de mantener un buen clima laboral manteniendo motivados al personal a través de incentivos, también se encarga de administrar los salarios a los empleados e intentar disminuir la rotación del personal, sin embargo, esta área es la encargada de realizar la contratación a los nuevos empleados, así como también de captar o realizar los reclutamientos y el despido de aquellos que no cumplen con las expectativas exigidas por la empresa. (Significados.com, 2017)

En líneas generales la administración de recursos humanos es la gestión del capital humano de una empresa, institución u organización, que gracias a las relaciones personales que existen entre los que la componen es que se logra el desempeño adecuado de los objetivos planteados por la organización. Por lo tanto, es de vital importancia mantener un análisis exhaustivo y permanente en la comunicación y el desempeño que existe entre el personal a modo de asegurar una efectiva gestión empresarial.

\section{Métodos y materiales}

Esta investigación, está enfocada en un análisis de la administración de recursos humanos como parte efectiva de la gestión empresarial y en el estudio de las relaciones interpersonales entre los empleados y la influencia que esto tiene sobre el desempeño de los objetivos generales y específicos de la organización.

La revisión se ha centrado en textos, documentos y artículos científicos publicados disponibles en la web, considerando que aquella herencia de la globalización nos permite acceder a mayor y mejor información a través de las herramientas tecnológicas. El motor de búsqueda ha sido herramientas académicas de la web que direccionan específicamente a archivos con validez y reconocimiento científico, descartando toda información no confirmada o sin las respectivas referencias bibliográficas.

\section{Resultados}

Las personas que conforman una empresa son el motor de empuje para conseguir el éxito en el cumpliendo de los objetivos que se plantean bien sea a corto, mediano o largo plazo, a través de 
las ideas que aportan los trabajadores es posible lograr y sacar adelante toda organización por muy pequeña o grande que esta pueda ser. Es vital que entre las personas exista un ambiente de empatía es decir un buen clima laboral, ya que, es por medio de la comunicación es que se logra el éxito en todos los ámbitos, personales y laboral.

La relación que existe entre todos los departamentos de una organización debe ser exitosa, los estudios demuestran que la alta gerencia y los directores de finanzas reconocen que la administración de recursos humanos llega a tener un papel crítico para el logro de las metas estratégicas de una empresa. La imagen 1, resume algunos resultados; indica, además, que ellos saben que el capital humano (los conocimientos, las habilidades y las experiencias de los empleados) puede tener gran efecto sobre los resultados organizacionales, como la satisfacción del cliente y la rentabilidad. (DESSLER, 2009, p. 47).

Imagen 1. Efectos que los directores de finanzas creen que el capital humano tiene sobre los resultados de negocios

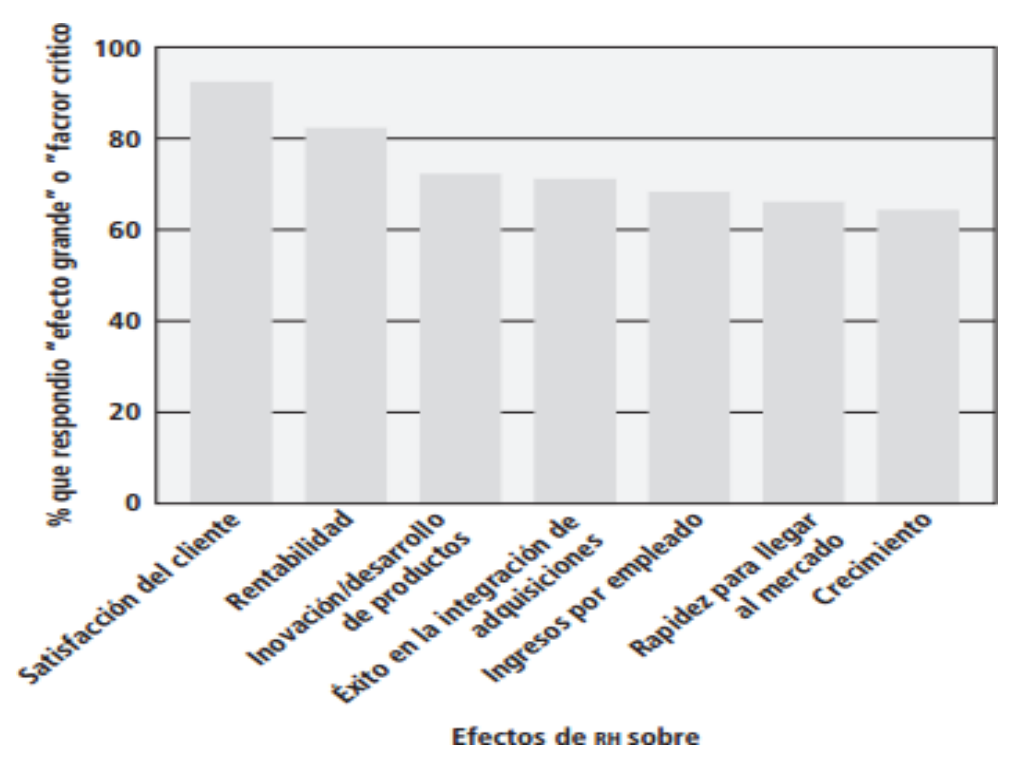

Fuente: (DESSLER, 2009, p. 47).

El entorno es parte del buen desarrollo que se tiene en la administración de recursos humanos, así como también las exigencias que existen por parte de las personas que hacen vida dentro de la 
organización, esto debido a que siempre se está expuesto a cambios a los cuales las empresas deben responder de la mejor manera, esto se logra a través de una gestión que se oriente a aumentar los niveles de competitividad en un mercado global.

La gestión de recursos humanos tiene que responder a una serie de exigencias del entorno, organizativas y exigencias individuales que se pueden representar como se muestra continuación según (Gómez- Mejía, 2001, p. 4), en donde se observa la relación que existe entre el entorno la organización y los individuos.

Imagen 2. Exigencias para los Recursos Humanos

\begin{tabular}{|c|} 
Entorno \\
Cambio rápido \\
Crecimiento de Internet \\
Globalización y diversidad de la fuerza de trabajo \\
Legislación \\
Evolución del trabajo y papel de la familia \\
$\begin{array}{c}\text { Organización } \\
\text { Posición Competitiva; Coste; Calidad } \\
\text { Flexibilidad empresarial } \\
\text { Reestructuración organizativa } \\
\text { Individuo } \\
\text { Ajuste persona-organización } \\
\text { Dilemas éticos y responsabilidad social } \\
\text { Delegación de responsabilidades } \\
\text { Inseguridad en el trabajo }\end{array}$ \\
\hline
\end{tabular}

Fuente: (Gómez- Mejía, 2001).

La efectiva gestión en la administración de recursos humanos, no solo está vinculada con el desempeño del personal, esta forma parte de los niveles estratégicos que se tomen para lograr la generación de recursos y capacidades valiosas que marquen la diferencia con respecto a las empresas de la competencia. La gestión de recursos humanos debe estar totalmente vinculada con 
la percepción e ideas de los empleados para poder optimizar y mantener el equilibrio entre los factores sociales y económicos.

Según un estudio realizado por Sopirla (2013, p 8), considerando como argumentada la necesidad de la gestión de recursos humanos en la empresa, a continuación, se propone repasar brevemente su evolución y argumentar el enfoque desde la perspectiva estratégica, para posteriormente tratar los principales procesos. Estos procesos están representados de manera esquemática en la imagen 3.

Imagen 3. Funciones y actividades en la Gestión de los Recursos Humanos.

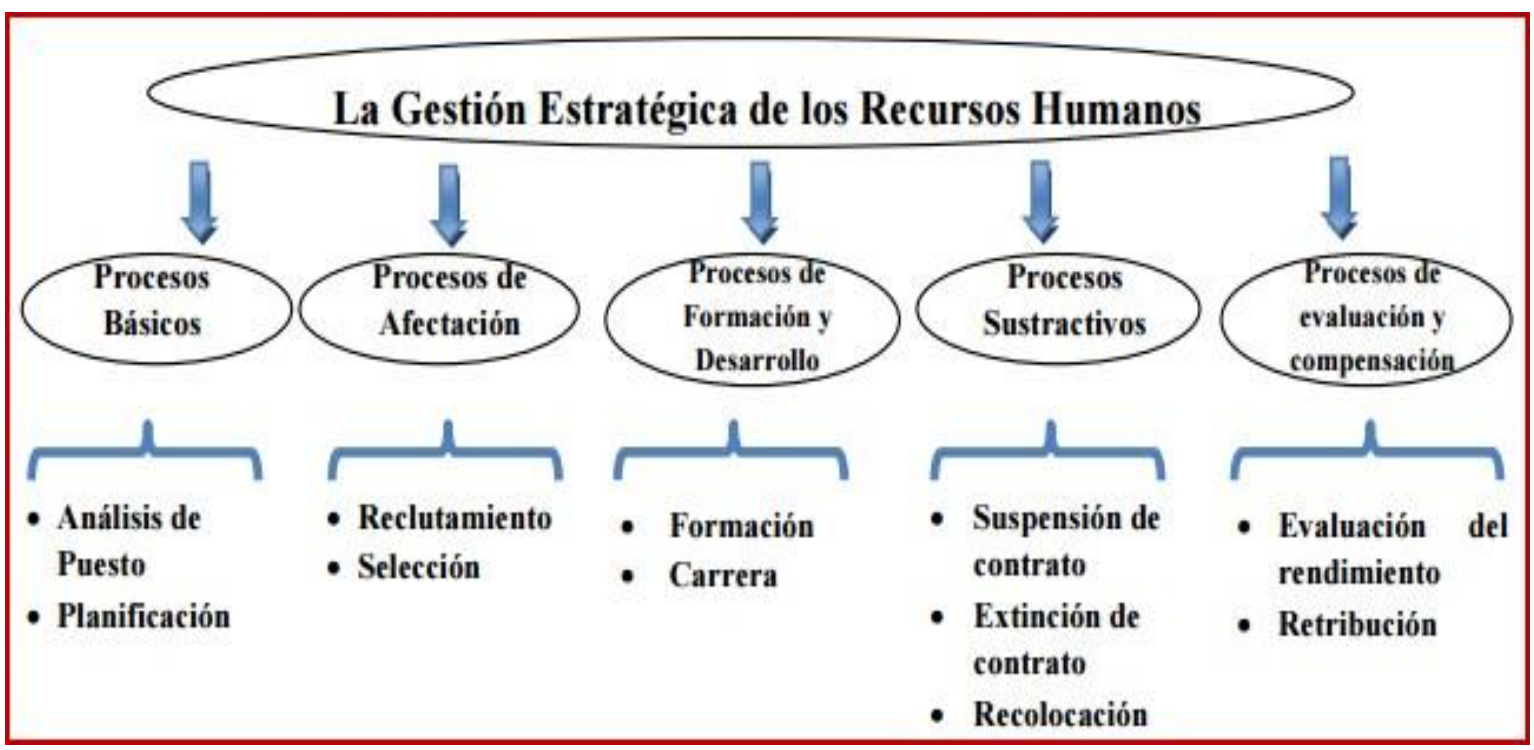

Fuente: (Sopirla, 2013).

Sin embargo, los esquemas y la estructura en la administración de recursos humanos han tenido sus cambios con el tiempo. A continuación, se presenta una tabla comparativa en donde se observa la reestructuración en esta área de la administración empresarial.

Tabla 1. Reestructuración de la función de Recursos Humanos

\begin{tabular}{|l|l|l|}
\hline Rol & Recursos Humanos Ayer & Recursos Humanos Hoy \\
\hline Político, centralizado & $\begin{array}{l}\text { Descentralizado; miembro } \\
\text { de los equipos directivos de } \\
\text { cada división }\end{array}$ \\
\hline
\end{tabular}




\begin{tabular}{|c|c|c|}
\hline $\begin{array}{l}\text { Captación y } \\
\text { selección de } \\
\text { personal }\end{array}$ & $\begin{array}{l}\text { Pone anuncios, dirige } \\
\text { entrevistas y chequea } \\
\text { referencias }\end{array}$ & $\begin{array}{l}\text { Predice requisitos futuros de } \\
\text { personal y capacidades que } \\
\text { respalden el plan estratégico. } \\
\text { Desarrolla programas para } \\
\text { ser un lugar atractivo en el } \\
\text { que trabajar. }\end{array}$ \\
\hline Retribución & $\begin{array}{l}\text { Transaccional y centrado } \\
\text { administrativamente. } \\
\text { Prácticas incoherentes } \\
\text { dentro de la empresa }\end{array}$ & $\begin{array}{l}\text { Diseña planes de actuación } \\
\text { equitativos que vinculan la } \\
\text { retribución con la actuación } \\
\text { divisional de la empresa. }\end{array}$ \\
\hline $\begin{array}{l}\text { Desarrollo } \\
\text { ejecutivo e } \\
\text { individual }\end{array}$ & $\begin{array}{l}\text { Informal y depende de } \\
\text { cada directivo }\end{array}$ & $\begin{array}{l}\text { Identificación de } \\
\text { competencias } \\
\text { organizacionales e } \\
\text { individuales clave que } \\
\text { respalden la empresa; planes } \\
\text { para contratarlas }\end{array}$ \\
\hline Empleado & Errático e Incoherente & $\begin{array}{l}\text { Planes de comunicación y } \\
\text { acción: visión, } \\
\text { valores planes }\end{array}$ \\
\hline $\begin{array}{l}\text { Políticas y } \\
\text { procedimientos }\end{array}$ & $\begin{array}{l}\text { Rígidas, pero se rompen } \\
\text { muchas reglas }\end{array}$ & $\begin{array}{l}\text { Líneas guía ligadas a } \\
\text { tendencias empresariales y } \\
\text { cuestiones emergentes. }\end{array}$ \\
\hline
\end{tabular}

Fuente: (G., 2004).

Haciendo un análisis de los resultados planteados en el trabajo de investigación, se tiene que llevar a cabo un estudio de las relaciones personales entre los empleados de una empresa brindar incentivos y procurar siempre a la armonía por parte de la gerencia es la pieza fundamental para el acorde desempeño de toda organización.

\section{Conclusiones}

Como hemos observado en el desarrollo del trabajo el personal que trabaja en una empresa, son los que hacen posible el crecimiento de la organización, son ellos quienes representan la parte más importante y quienes logran que sea posible satisfacer las necesidades de los clientes y mantener un ambiente totalmente productivo con la finalidad de cumplir los objetivos generales y específicos y de superar las expectativas y metas dispuestas por la organización. 
En el trabajo se observó que, mientras exista una relación exitosa dentro de los departamentos se incrementan los resultados organizacionales teniendo la administración de los recursos humanos un papel crítico para el logro de las metas estratégicas de una empresa aumentando la satisfacción de los clientes y la rentabilidad.

En el trabajo también se observó que, el entorno influye sobre la gestión de los recursos humanos, que las empresas deben estar preparadas para los cambios que se generen y que tanto como el entorno, la organización y el individuo están interrelacionados por lo que las empresas deben tener en cuenta ya que esto podría llegar a afectar a la organización en el cierto caso de que un empleado tenga un problema y no sea expresado a tiempo.

Se observó, que la gestión de los recursos humanos está vinculada con las ideas en planes y estrategias que planteen los trabajadores que, así como también se encarga de mantener la armonía en el ambiente laboral, es también importante que los trabajadores sean tomados en cuenta a modo de ofrecer ideas que ayuden al crecimiento empresarial. Es por esto, que la gestión estratégica de los recursos humanos está relacionada con todos los procesos referentes al personal desde los procesos básicos, hasta las evaluaciones y compensaciones, en donde se reconoce el esfuerzo de los empleados.

Finalmente, se puede concluir que la estructura de la administración de recursos humanos, ha tenidos cambios con el paso de los años, enmarcados a buscar una mayor relación entre los departamentos, para brindar mejoras a la empresa, así como también el mayor cumpliendo de sus objetivos y una mayor rentabilidad comercial.

\section{Referencias Bibliográficas}

Chiavenato, I. V. (2007). Administración de recursos humanos El capital humano de las organizaciones.

DESSLER, G. (2009). Administración de recursos humanos.

G., M. S. (Enero de 2004). Administración de recursos humanos ¿Cómo funciona? Obtenido de https://www.gestiopolis.com/administracion-de-recursos-humanos-como-funciona/

Gómez- Mejía, L. B. (2001). Dirección y gestión de recursos humanos. 3 ed. Prentice Hall. Madrid. 
Peña,

$\mathrm{k}$.

(2012).

Administracion4u.

Obtenido

de

http://administracion4u.blogspot.com/2012/04/ramas-de-la-administracion.html

Significados.com. (2017). Obtenido de https://www.significados.com/administracion-de-recursoshumanos/

Sopirla, D. C. (2013). Plan Estratégico de Gestión de Recursos Humanos. 